\title{
Evidence for an Anisotropic State of Two-Dimensional Electrons in High Landau Levels
}

\author{
M. P. Lilly, ${ }^{1}$ K. B. Cooper,${ }^{1}$ J.P. Eisenstein, ${ }^{1}$ L. N. Pfeiffer, ${ }^{2}$ and K. W. West ${ }^{2}$ \\ ${ }^{1}$ California Institute of Technology, Pasadena, California 91125 \\ ${ }^{2}$ Bell Laboratories, Lucent Technologies, Murray Hill, New Jersey 07974
}

(Received 19 August 1998)

\begin{abstract}
Magnetotransport experiments on high mobility two-dimensional electron gases in GaAs/AlGaAs heterostructures have revealed striking anomalies near half filling of several spin-resolved, yet highly excited, Landau levels. These anomalies include strong anisotropies and nonlinearities of the longitudinal resistivity $\rho_{x x}$ which commence only below about $150 \mathrm{mK}$. These phenomena are not seen in the ground state or first excited Landau level but begin abruptly in the third level. Although their origin remains unclear, we speculate that they reflect the spontaneous development of a generic anisotropic many-electron state. [S0031-9007(98)08178-2]
\end{abstract}

PACS numbers: 73.20.Dx, 73.40.Kp, 73.50.Jt

A magnetic field applied perpendicular to the plane of a two-dimensional electron gas (2DEG) resolves the energy spectrum into discrete Landau levels (LLs). As the field increases, the Fermi level drops down through the Landau ladder in a series of steps until, at high field, it resides in the lowest $(N=0)$ level. In this situation the kinetic energy of the electrons is quenched and electron-electron interactions dominate the physics with the fractional quantized Hall effect (FQHE) as the most spectacular consequence [1]. After more than 15 years of study, much is known about electron correlations in this lowest LL case. The same cannot be said when the Fermi level is in a higher Landau level. In the second LL $(N=1)$, the FQHE is virtually absent; only fragile and poorly understood states at Landau filling fractions $\nu=7 / 3,5 / 2$, and $8 / 3$ are seen in the best samples. In the third and higher LLs $(N \geq 2)$ still less is known, although there have been interesting suggestions of charge density waves in the clean limit $[2,3]$. At very high $N$, and therefore very low magnetic field, the Landau level splitting becomes insignificant and the 2DEG assumes the character of a weakly disordered Fermi liquid.

In this paper we report the observation of several dramatic anomalies in the low temperature magnetotransport of clean 2DEGs when the Fermi level lies near the middle of a spin-resolved highly excited Landau level. These effects, which commence only below about $150 \mathrm{mK}$, abruptly begin and are strongest in the third $(N=2) \mathrm{LL}$, but persist up to about $N=6$. Including strong anisotropies and intriguing nonlinearities of the resistivity, these effects suggest a considerably more interesting tableau at high $N$ than independent electrons moving in a disordered Landau band.

The samples used in this study are GaAs/AlGaAs heterojunctions grown by molecular beam epitaxy (MBE). Data from six samples ( $A$ through $F$ ) will be discussed. Samples $A, B$, and $C$ were taken from one MBE wafer, $D$ and $E$ from a second, and $F$ from a third. Each wafer was rotated during growth to ensure high homogeneity of the electron density $n_{s}$. These densities (in units of $10^{11} \mathrm{~cm}^{-2}$ ) are close to $n_{s}=2.67$ for samples $A, B$, and
$C ; n_{s}=2.27$ for samples $D$ and $E$; and $n_{s}=1.52$ for sample $F$. The low temperature mobility of each is $\mu \geq$ $9 \times 10^{6} \mathrm{~cm}^{2} / \mathrm{V} \mathrm{s}$. Each sample was cleaved (along $\langle 110\rangle$ directions) into a $5 \times 5 \mathrm{~mm}$ square from its parent $\langle 001\rangle$ wafer. For samples $A, D, E$, and $F$, indium contacts were placed at the corners and the midpoints of the sides of the chip. Hall bar patterns were lithographically etched onto samples $B$ and $C$ before the contacts were made. The samples were briefly illuminated at low temperature with a red LED. Electrical transport measurements were performed using 2-20 nA, $13 \mathrm{~Hz}$ excitation, although for the nonlinearity studies an additional dc current was imposed.

Figure 1 shows the resistivity [4] $\rho_{x x}$ at $T=150 \mathrm{mk}$ of sample $A$. Shubnikov-deHaas ( $\mathrm{SdH}$ ) oscillations commence at around $B \approx 60 \mathrm{mT}$ and the spin splitting of the Landau levels is evident by $130 \mathrm{mT}$. The smallness of these fields attests to the high quality of the 2DEG in this sample. Between about $B=5.5$ and $11 \mathrm{~T}$ the Fermi level is in the upper spin branch of the $N=0$ lowest LL. This corresponds to Landau level filling fractions $\nu=h n_{s} / e B$ between $\nu=2$ and $\nu=1$. Clear signatures

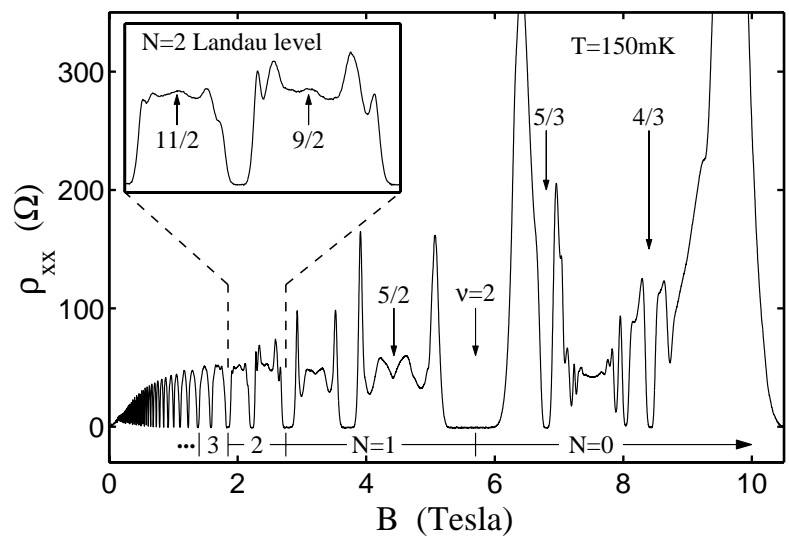

FIG. 1. Overview of diagonal resistivity in sample $A$ at $T=$ $150 \mathrm{mk}$. Structure in the $N=2$ Landau level is expanded in the inset. 


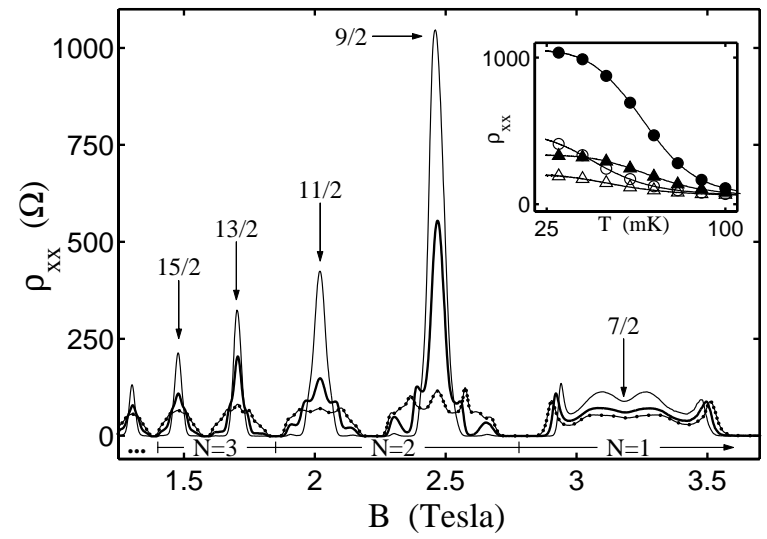

FIG. 2. Peaks in $\rho_{x x}$ in sample $A$ developing at low temperatures in high LLs (dotted line: $T=100 \mathrm{mK}$; thick line: $65 \mathrm{mK}$; thin line: $25 \mathrm{mK}$ ). Inset: temperature dependence of peak height at $\nu=9 / 2$ (closed circles), 11/2 (open circles), $13 / 2$ (closed triangles), and 15/2 (open triangles).

of several fractional quantized Hall states are evident. Between $B=2.8$ and $5.5 \mathrm{~T}$ the Fermi level is in the $N=1$ second LL and the developing $\nu=5 / 2 \mathrm{FQHE}$ is indicated in the figure. At lower temperatures this state, and the nearby $\nu=7 / 3$ state, strengthen. In the upper spin branch of this same LL a minimum in $\rho_{x x}$ is seen at $\nu=7 / 2$, but it does not deepen as the temperature is reduced.

Below $B \approx 2.8 \mathrm{~T}$ the Fermi level is in the $N=2$ and higher LLs. The inset to Fig. 1 reveals that in both spin branches of the $N=2 \mathrm{LL}$ there are a number of maxima and minima in $\rho_{x x}$. Although this complex structure does not appear to be associated with a FQHE (no quantized plateaus in the Hall resistivity $\rho_{x y}$ have been found), its existence strongly indicates that electron correlations are important. In the standard model [5], if disorder overwhelms interactions, a simple peak in the resistivity separates the broad zeros of adjacent integer quantum Hall states.

Figure 2 displays the temperature dependence of the $\rho_{x x}$ features in the $N=1, N=2$, and $N=3$ LLs in sample $A$. Below $T=150 \mathrm{mk}$ peaks develop at $\nu=$ $9 / 2,11 / 2,13 / 2$, and $15 / 2$ which grow rapidly below $100 \mathrm{mK}$; by $25 \mathrm{mK}$ the peak at $\nu=9 / 2$ has exceeded $1000 \Omega$. Surprisingly, the peaks do not narrow as $T$ is reduced. The subsidiary structures flanking the peaks do fall with temperature but are not simply "consumed" by the widening of the nearby integer QHE states; even at $25 \mathrm{mK}$ they are still evident [6]. This behavior does not fit the standard model of disorder-driven integer QHE transitions, but suggests instead that interactions remain important down to very low $T$. The observed behavior of $\rho_{x x}$ in the $N=1$ LL (only the upper spin branch, $4>$ $\nu>3$, is shown in the figure) is qualitatively different. Instead of peaks, there are minima at $\nu=7 / 2$ and at the fragile fractional QHE state at $\nu=5 / 2$. The closeness in magnetic field of the $\nu=9 / 2$ and $7 / 2$ filling factors makes this difference particularly striking. This basic

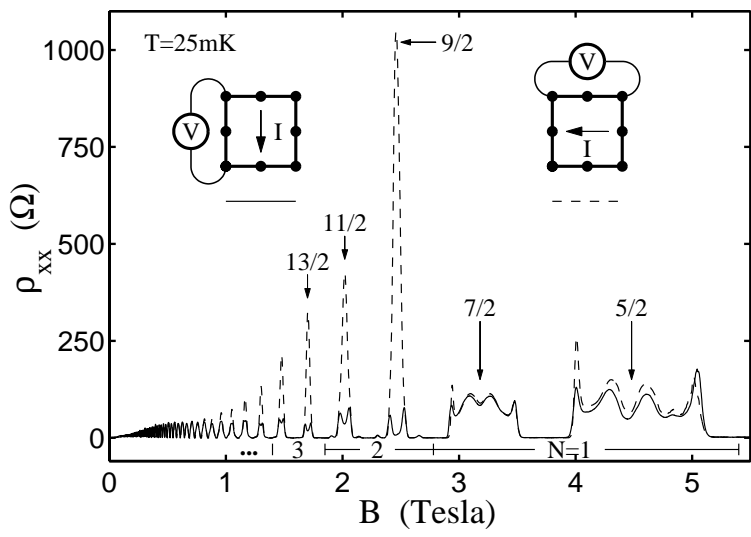

FIG. 3. Anisotropy of $\rho_{x x}$ in sample $A$ at $T=25 \mathrm{mK}$. The two traces result from simply changing the direction of current through the sample; the sample itself is not rotated.

result is just as clearly evident in samples $B$ through $F$. In the inset to Fig. $2, \rho_{x x}$ at $\nu=9 / 2,11 / 2,13 / 2$, and $15 / 2$ is plotted versus temperature.

Figure 3 displays our most remarkable finding. The two traces are resistances measured in sample $A$ at $T=$ $25 \mathrm{mk}$ for two perpendicular directions of the current flow through the sample. The diagrams in the figure depict the difference between the two configurations. This seemingly innocuous change vastly alters the resistivity in the $N=2$ and several higher LLs. Note that the solid curve has been multiplied by a factor of 0.62 in order to match the data sets in the very low field regime. A variation of this size in the resistance of a quantum Hall sample is quite common and may simply reflect irregularities in the positions of the contacts. In any case, this factor in no way obscures the dramatic anisotropy of the resistivity near the centers of both spin branches of the $N=2$ through $N=5$ LLs. While peaks in $\rho_{x x}$ are evident in one configuration, relatively deep minima are seen in the other [7]. At $\nu=9 / 2$ the ratio of the resistances is close to 100 . Equally striking is the fact that no comparable anisotropy is apparent in the $N=$ $1 \mathrm{LL}$ (nor the $N=0$ level which is not shown in the figure). The anisotropy we are reporting is apparently confined to the centers of the LLs; well away from half filling (of each spin branch) the two resistances again roughly match (after the scaling factor of 0.62 is applied). As the temperature is increased, the anisotropy in $\rho_{x x}$ subsides until by $T=150 \mathrm{mK}$ it is no longer significant. We emphasize that for no current configuration are any plateaus seen in the Hall resistance $\rho_{x y}$ at half filling of the $N \geq 2$ Landau levels.

By measuring $\rho_{x x}$ with various contact configurations we have determined that the orientation of the anisotropy is fixed within the sample and is insensitive to reversal of the magnetic field and thermal cycling to room temperature. Although the geometry of sample $A$ is quite open and the precise current distribution is unknown, our data suggest that the "principal axes" of the anisotropy are 


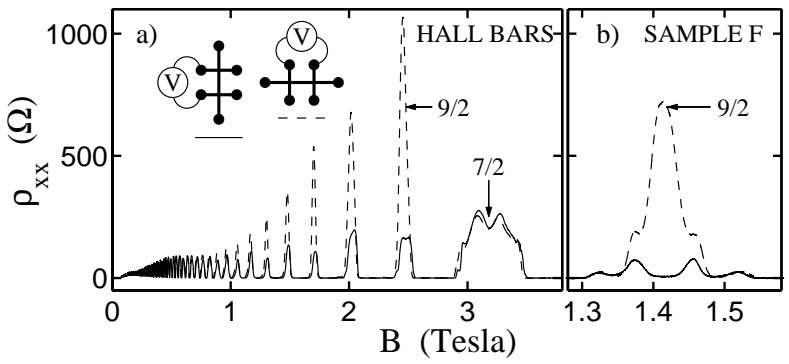

FIG. 4. (a) Anisotropy of $\rho_{x x}$ at $T=25 \mathrm{mK}$ measured using Hall bar samples $B$ (dashed line) and $C$ (solid line). (b) Anisotropy of $\rho_{x x}$ at $T=15 \mathrm{mK}$ measured in the low density sample $F$. Current flow configurations as in Fig. 3.

roughly parallel to the sides of the chip. In order to better define the current path, Hall bar samples $B$ and $C$ were examined. In sample $B$ the bar axis was oriented parallel to the $\langle 1 \overline{1} 0\rangle$ crystal axis, while in sample $C$ the bar was aligned along $\langle 110\rangle$. Figure $4 \mathrm{a}$ compares $\rho_{x x}$ data from these two samples. At $\nu=9 / 2$ the two bars exhibit a large (roughly sixfold) anisotropy in $\rho_{x x}$ while at $\nu=7 / 2$ in the $N=1 \mathrm{LL}$ the observed $\rho_{x x}$ is isotropic. (The data from Hall bar $C$ were multiplied by a factor of 0.75 to match the amplitude of its $\mathrm{SdH}$ oscillations at very low field with those of Hall bar B.) Though not shown in the figure, isotropy is also observed at $\nu=5 / 2$ and $3 / 2$. As in sample $A$, substantial anisotropy exists near $\nu=11 / 2,13 / 2,15 / 2$, and $17 / 2$ before isotropy returns at low magnetic field. The orientation of the anisotropy in sample $A$ agrees with that seen in the Hall bars. While the magnitude of the $\nu=9 / 2$ anisotropy in the Hall bars is weaker than that seen in the sample $A$, it seems clear that the same basic effect is at work. Finally, the anisotropy effect is also seen in the lower density samples $D$ and $F$, which come from different MBE wafers. Figure $4 \mathrm{~b}$ shows data from sample $F$ in which the $9 / 2$ anisotropy is nearly 100-fold even after magnifying the data from the $\rho_{x x}$-minimum configuration by a factor of 1.6 to match the low field $\mathrm{SdH}$ oscillations.

The sudden development at very low temperatures of strong temperature dependences and large anisotropies of the resistivity of clean 2DEGs in the third and several higher Landau levels suggests that some previously unappreciated physics is at work. The anisotropy is intriguing as it is not at all clear what breaks the in-plane symmetry of the system. There are various extrinsic effects related to the MBE growth that might pick out a direction in the 2D plane. A wafer-scale gradient in the electron density due to the off-normal positions of the various elemental sources in the MBE chamber is one possibility. However, our samples were rotated during their growth specifically to minimize such gradients. Indeed, measurements of the $\mathrm{SdH}$ periodicity using many different voltage and current configurations (including those which produce the large anisotropy displayed in Fig. 3) show no more than a $0.3 \%$ variation. This result strongly suggests that there is at most a very small density gradient. At the same time, the low onset field of the $\mathrm{SdH}$ oscillations $(B \approx 60 \mathrm{mT}$, corresponding to Landau filling fraction $\nu \approx 180$ ) seen in all contact configurations suggests that density fluctuations on short distance scales are also small. Among other possible extrinsic symmetry-breaking effects, we mention the often observed "slip lines" which are believed to be steps on the surface of MBE wafers, and the possibility that the $\langle 001\rangle \mathrm{GaAs}$ substrate was slightly miscut from its parent boule.

It is possible that the observed anisotropy in $\rho_{x x}$ is due to some unforeseen order in the static disorder potential. This is unlikely since the anisotropy is seen only in the $N=2$ and a handful of higher LLs. It is not present in the lowest or first excited LL, nor is it seen in the semiclassical regime well below $B=1 \mathrm{~T}$. The effects reported here are, however, reminiscent of recently observed transport anisotropies near $\nu=1 / 2$ in the lowest LL in samples where a periodic density modulation has been externally imposed [8,9]. A theoretical basis for understanding this effect has been proposed [10], and it may turn out to be useful in the present context [11], although it would not identify the source of the inhomogeneity.

Recently, Koulakov, Fogler, and Shklovskii [2] and subsequently Moessner and Chalker [3] have proposed that in a clean 2DEG in the $N=2$ and higher LLs the uniform electron liquid may be unstable against the formation of charge density waves (CDW). They further suggest that near half filling of the LL the CDW is a unidirectional "stripe phase" having a wavelength of order the cyclotron radius. In this stripe phase the electron density in the uppermost LL alternates between zero and full filling. At $\nu=9 / 2$ this implies there are stripes of the incompressible QHE states $\nu=4$ and $\nu=5$. While it is surely plausible that electrical transport in such a unidirectional phase would be anisotropic, it is not clear what would pin the stripes or why they are apparently coherent over the macroscopic size of our samples.

To further investigate magnetotransport in highly excited LLs, we have examined the linearity of $\rho_{x x}$. Figure 5 summarizes our $T=25 \mathrm{mK}$ results from sample $E$. The figure displays the differential resistivity $d V_{x x} / d I$ at $\nu=9 / 2,11 / 2$, etc., measured using $5 \mathrm{nA}, 13 \mathrm{~Hz}$ excitation, in the presence of an added dc current $I_{\mathrm{dc}}$. The data were taken using a contact configuration for which the resistivity (i.e., $d V_{x x} / d I$ at $I_{\mathrm{dc}}=0$ ) exhibits strong peaks at these filling factors. In each panel $I_{\mathrm{dc}}$ runs from -200 to $+200 \mathrm{nA}$, and the plotted $d V_{x x} / d I$ data are normalized by their value at $I_{\mathrm{dc}}=0$. Several of the data sets show marked nonlinearity. At $\nu=9 / 2$, where the effect is strongest, $d V_{x x} / d I$ at first increases substantially as $I_{\mathrm{dc}}$ is applied. At about $\pm 100 \mathrm{nA}$ it reaches a maximum and then falls off at higher current. Since $\rho_{x x}$ at $\nu=9 / 2$ falls with rising temperature, it is clear that the initial rise in $d V_{x x} / d I$ at small $I_{\mathrm{dc}}$ is not consistent with electron 


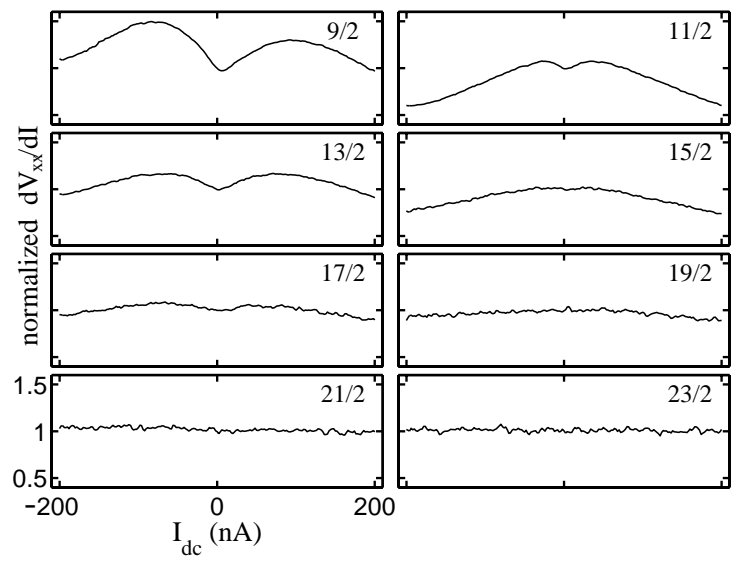

FIG. 5. Nonlinearity of differential resistivity $d V_{x x} / d I$ in sample $C$ at half filling of several high LLs at $T=25 \mathrm{mK}$. The resistivity is normalized by its value at $I_{\mathrm{dc}}=0$. In each panel $I_{\mathrm{dc}}$ runs from -200 to $+200 \mathrm{nA}$ and the normalized $d V_{x x} / d I$ from 0.5 to 1.5 .

heating. At large $I_{\mathrm{dc}}$ the falling of $d V_{x x} / d I$ may indeed be due to heating. Qualitatively similar nonlinearities are seen at half filling of the higher LLs, but the effect eventually dies away at low magnetic field. Note the oscillatory strength of the nonlinearity: strong at $9 / 2$, weaker at $11 / 2$, relatively stronger at $13 / 2$, weak again at $15 / 2$, and so on. As with the anisotropy, the nonlinearity is a low temperature phenomenon; by $150 \mathrm{mK}$ it is essentially gone. It is more fragile than the anisotropy; while clearly evident in some samples it is weaker in others. We emphasize that in each LL the nonlinearity is seen over a range of $\nu$ around half filling. Finally, although weak nonlinearities have sometimes been seen in the $N=1$ and $N=0 \mathrm{LL}$, they appear qualitatively different from that seen in the $N=2$ and higher LLs.

The increasing differential resistivity for small $\mathrm{dc}$ currents also implies an increasing conductivity. This follows from inverting the resistivity tensor and noting that the Hall resistivity $\rho_{x y}$ is several times larger than $\rho_{x x}$ over the field range of interest. Although an increasing conductivity is suggestive of a depinning mechanism, we have seen no evidence of a sharp threshold; the nonlinearity appears to turn on continuously.

In conclusion, we have reported dramatic magnetotransport anomalies that are specific to the third and higher
Landau levels in clean 2DEG's in GaAs/AlGaAs heterostructures. These effects, which appear only at low temperature, include huge anisotropies in the resistivity near half filling of the spin-resolved Landau levels as well as intriguing nonlinearities. The origin of these effects is unclear, but the data are consistent with the spontaneous development of an anisotropic electronic configuration. The occurrence of the same basic transport signatures in several adjacent highly excited Landau levels points to a generic mechanism.

We thank J. Chalker, S. M. Girvin, B. Halperin, A. H. MacDonald, and K. Yang for helpful discussions and Ian Spielman for help in determining the crystallographic axes in our samples. This work was supported by the National Science Foundation.

[1] For recent reviews, see Perspectives in Quantum Hall Effects, edited by S. Das Sarma and A. Pinczuk (John Wiley, New York, 1997).

[2] A. A. Koulakov, M. M. Fogler, and B. I. Shklovskii, Phys. Rev. Lett. 76, 499 (1996); Phys. Rev. B 54, 1853 (1996); M. M. Fogler and A. A. Koulakov, Phys. Rev. B 55, 9326 (1997).

[3] R. Moessner and J. T. Chalker, Phys. Rev. B 54, 5006 (1996).

[4] As is commonplace, we employ the term resistivity even though the actual measured quantity is resistance; in square samples the factor relating the two is of order unity.

[5] See, for example, the chapter by S. Das Sarma in Ref. [1].

[6] At very low $T$ we find tiny peaks in $\rho_{x x}$ separated from the main peak at $\nu=9 / 2$ and $\nu=11 / 2$ by narrow regions in which $\rho_{x x} \approx 0$. We find that in these regions the Hall resistance is quantized, but at the value of the nearby integer QHE.

[7] In the $N=2 \mathrm{LL}$, this observation was, to our knowledge, first made by R. L. Willett and J.P. Eisenstein (unpublished) and has also been reported by H. L. Stormer et al., Bull. Am. Phys. Soc. 38, 235 (1993).

[8] R. L. Willett, K. W. West, and L. N. Pfeiffer, Phys. Rev. Lett. 78, 4478 (1997).

[9] J. Smet, K. von Klitzing, D. Weiss, and W. Wegscheider, Phys. Rev. Lett. 80, 4538 (1998).

[10] Felix von Oppen, Ady Stern, and Bertrand I. Halperin, Phys. Rev. Lett. 80, 4494 (1998).

[11] B. Halperin (private communication). 